\title{
Shape factor of relay response curves and its use in autotuning
}

\author{
R.C. Panda ${ }^{a}$, Cheng-Ching Yu ${ }^{\text {b,* }}$ \\ a Department of Chemical Engineering, CLRI (CSIR), Chennai 600 020, India \\ b Department of Chemical Engineering, National Taiwan University, 1, Roosevelt Rd., Sec. 4 Taipei 106-17, Taiwan
}

Received 26 July 2004; received in revised form 21 January 2005; accepted 21 February 2005

\begin{abstract}
Identification of process parameters using single relay feedback test is mostly used in practice. Limit cycle data along with shape factor of the response curves are important to identify the correct model structure and corresponding model parameters. Time domain analytical expressions are helpful in deriving conditions to estimate process parameters accurately. Second order plus dead time (SOPDT) processes are utilized to represent all different shapes with minimal number of model parameters. Provided with analytical expressions for relay feedback responses, identification algorithms are formulated and three different model structures are categorized. The autotuning procedure consists of the following steps. First, a relay feedback test is conducted and relay response is recorded for analysis. If the response is not symmetric we do a biased relay test to restore the symmetry, otherwise, we proceed for system identification. After finding out model structures and parameters suitable tuning rules are suggested for different ranges of dead time to time constant ratio $(D / \tau)$ and damping coefficient $(\xi)$ values. Closed-loop performances of the identified systems with and without measurement noise are found to be satisfactory.
\end{abstract}

(c) 2005 Elsevier Ltd. All rights reserved.

Keywords: Relay feedback; Modeling; Identification; Tuning; Disturbance rejection

\section{Introduction}

Autotuning using relay feedback is widely used in industry because of its simplicity. It is used to find dynamic information of the process and thereby to tune the feedback controller. The reliability of the method is made used of by Astrom and Hagglund [1] for PID controllers. Process identification using autotuning variation (ATV) is one useful tool today in process industries. Luyben [9] is one of the pioneers to explain ATV technique for system identification of low order processes in which ultimate gain and ultimate frequency are obtained (Astrom's autotuning method). Process

\footnotetext{
${ }^{*}$ Corresponding author. Address: Department of Chemical Engineering, National Taiwan University, 1, Roosevelt Rd., Sec. 4 Taipei 106-17, Taiwan. Tel.: +88623365 1759; fax: +88623366 3037 .

E-mail address: ccyu@ntu.edu.tw (C.-C. Yu).
}

model parameters were obtained from relay response of the system. Li et al. [8] proposed a method to estimate process gain by using information from two relay experiments. Chang et al. [3] derived transfer functions of first order plus dead time (FOPDT) systems from relay feedback tests with increased accuracy using autotuning variation (ATV) method. But these methods use frequency domain parameters $\left(K_{\mathrm{u}}\right.$ and $\left.P_{\mathrm{u}}\right)$, which are derived from describing functions and carry only approximate information of process at ultimate frequency. Few articles have been reported to find exact parameter estimation of low order transfer functions using single relay test $[15,17,7]$ or by using A-locus method [12,5]. But closed-form solutions are not available for general transfer functions. Progress in relay feedback is summarized by $\mathrm{Yu}$ [18]. Most of the literature discusses identification of process dynamics using initial part of the relay feedback (RFB) responses. But in practice it is difficult to store the very initial part of RFB response. So, 
effort has been made in this work to extract unknown process parameters from stabilized part of the RFB response. Moreover, estimation techniques using frequency domain ultimate properties do not yield very accurate process parameters and hence tuning gives a poor controller performance. Therefore, there is a need to identify exact parameters of the process. Mathematical models of relay feedback responses in time domain are helpful to estimate exact process parameters. Recently, Luyben [10] discussed a very simple technique that needs one additional parameter (other than limit cycle data), namely, shape factor, to identify process transfer functions using a single relay feedback test for stable and unstable FOPDT systems. The shapes of relay response curves primarily give an idea of the system category and its order. Thyagarajan and $\mathrm{Yu}$ [16] categorized process models by observing the shapes of relay feedback response (generated from mostly FOPDT processes with different $D / \tau$ ratio and higher order systems) and identified the transfer function models.

The purpose of this work is to utilize the shape information from the relay feedback test to identify the correct model structure of the process and to calculate appropriate PID controller settings. This paper is organized as follows: an introduction to relay feedback,

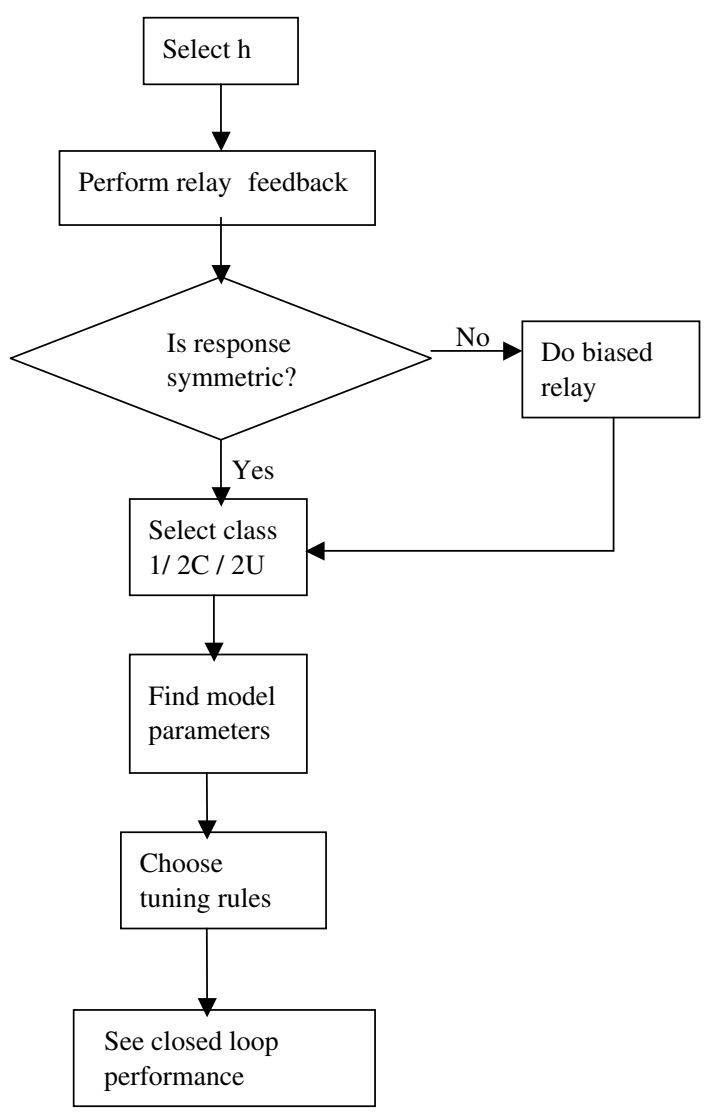

Fig. 1. Flow chart for the proposed scheme of system identification through relay feed back. observation related to process dynamics made from different shapes and categorization of different model structures based on multiplicative error analysis are presented in Section 2. The identification of model parameters is explained in Section 3. Section 4 deals with retention of shapes in spite of load disturbances. Calculation of tuning parameters and implementation of PID controller to the actual process is described in Section 5. Concluding remarks are drawn at the end. Proposed scheme of identification is shown in Fig. 1.

\section{Relay feedback response and shapes}

\subsection{Relay feedback}

Astrom and Hagglund [1] proposed that if a relay of magnitude ' $h$ ' is inserted in a feedback loop, the input $u(t)$ becomes ' $h$ '. If the relay output lags behind the input by $\pi$ radians, the closed-loop system starts oscillating around set-point with a period of $P_{\mathrm{u}}$ (Fig. 2). As the output $y(t)$ starts increasing after a time delay of ' $D$ ', the relay output switches to opposite direction and becomes $u(t)=-h$. With a phase lag of $-\pi$, a limit cycle of amplitude ' $a$ ' is formed and the process variable crosses the set-point. From the principle harmonic approximation of the oscillations, the ultimate gain $\left(K_{\mathrm{u}}\right)$ can be approximated [1] as $K_{\mathrm{u}}=4 h / \pi a$ and ultimate frequency $\left(\omega_{\mathrm{u}}\right)$ thus becomes $\omega_{\mathrm{u}}=2 \pi / P_{\mathrm{u}}$ (where $P_{\mathrm{u}}$ is the period of oscillation). With the help of these two identified frequency domain parameters, $\omega_{\mathrm{u}}$ and $P_{\mathrm{u}}$, many tuning rules are formulated. Unfortunately, satisfactory closed loop performance is not always guaranteed as no single tuning rule is appropriate throughout a wide range of process delay $(D)$ to process time constant $(\tau)$ ratios $(D / \tau)$. We consider here different SOPDT systems (as they are rich in different dynamics) with different time
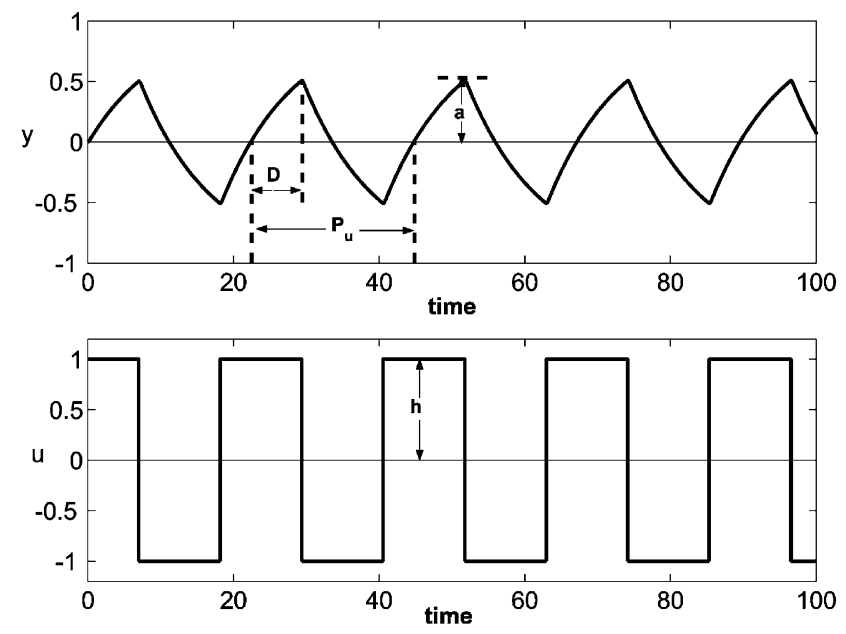

Fig. 2. Typical relay feed back output and input. 


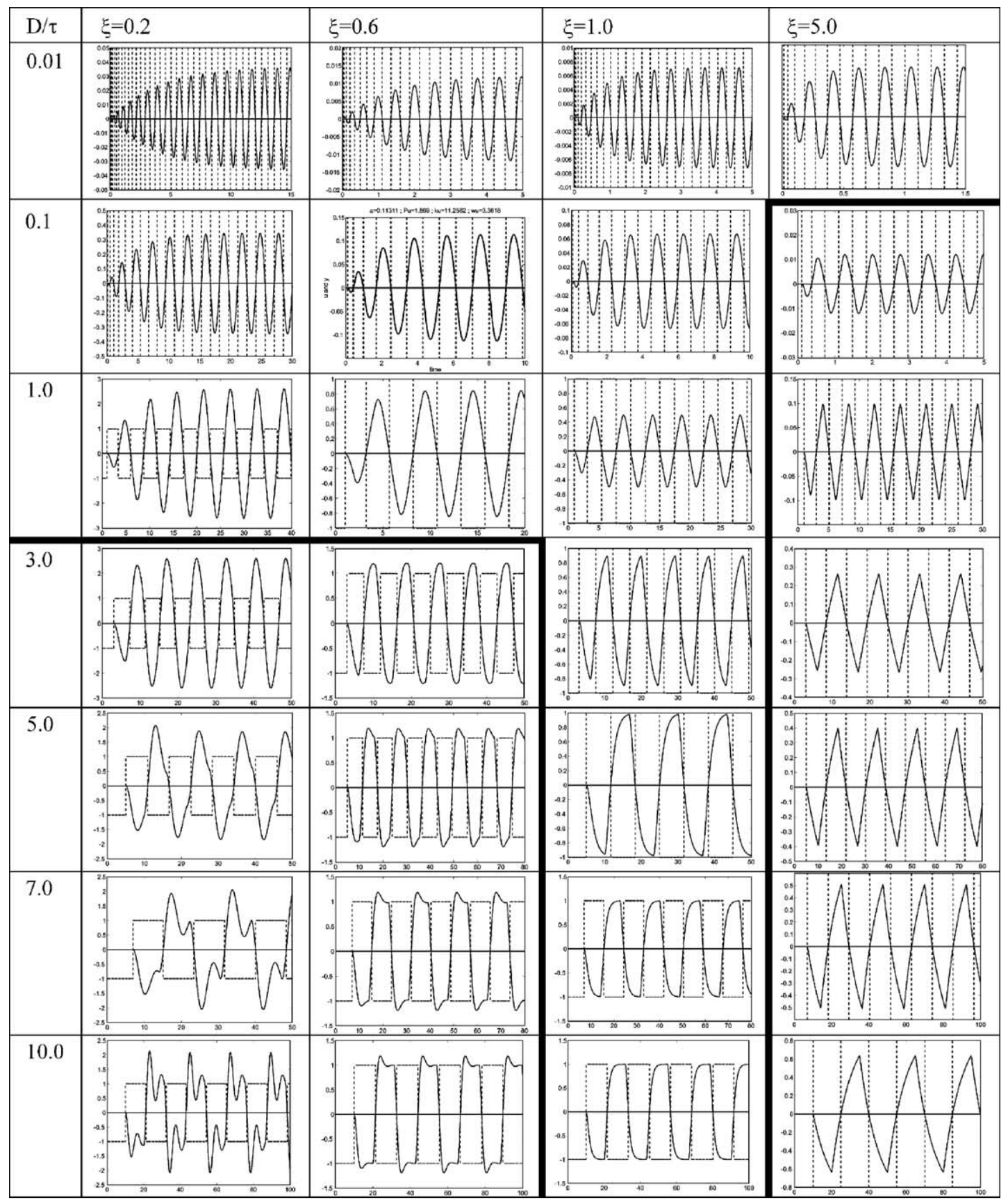

Fig. 3. Relay feedback responses for some SOPDT processes with different $D / \tau$ and $\xi$ values (thick line refers the classification boundary).

delay to time constant ratios $(D / \tau=0.01-10.0)$ and different damping coefficients $(\xi=0.2-5.0)$ values to study shapes (Fig. 3). Note that all the processes gains are assumed to be 1 and input relay height used is of $h=1$.

\subsection{Shapes}

Relay response of processes with increasing $\xi$ (column-wise) and increasing $D / \tau$ (row-wise) are recorded. A close look to Fig. 3 reveals that, there are generally three types of shapes in the sustained oscillations.
Firstly, with low $D / \tau$ ratio (processes with dominant time constant compared to the dead time) the shapes of the oscillations are symmetric with rounded peaks. The peaks of the oscillations form a smooth global curvature (similar to gradually developed step response) until it stabilizes. They look like exponentially developed cycles. As the $D / \tau$ ratio increases, and with damping coefficient less than 1 , the curvature disappears and the individual peaks of the oscillations become more curved. Secondly, with high $D / \tau$ ratio (more than 3.0) the relay response show typical under damped 
oscillations. Third and lastly, with $D / \tau>1$ and $\xi>1$, the shapes look fully developed with sharp edge at peak amplitude and takes somewhat rectangular shape.

Next, let us look at the effects of $D / \tau$ and $\xi$ on curve shapes. The time constant dominated processes show triangular with sharp or rounded peaks. With increase in both of dead time to time constant ratios $(D / \tau)$ and damping factor $(\xi)$, curvature begins to appear which implies gradual development toward a step response. As $D / \tau$ further increases, the response slowly becomes a symmetrical rectangular wave. But typical underdamped types of oscillations are observed with low $\xi$ and high $D / \tau$ value.

\subsection{Classification}

In control-relevant identification, it is important to identify correct model structure and, subsequently, appropriate tuning rules can be applied. Obviously, we would like to have a minimal number of model structures while capturing essential characteristics (shapes) in a relay feedback test. With above observations of shapes, three different classes are identified. These classifications are based on accommodating all possible types of curve shapes in relay feedback responses.

\subsubsection{Model structure 1 ( $M S-1$ )}

Shapes under third observation may be under first category, because, they look similar to the relay response produced by FOPDT systems. System under this category has following model structure (MS-1) (lower right corner in Fig. 3):

$G_{\mathrm{P}}(s)=\frac{K_{\mathrm{P}} \mathrm{e}^{-D s}}{\tau s+1}$

where $K_{\mathrm{P}}$ is the steady state gain, $D$ is the time delay, and $\tau$ is the process time constant. Systems with large $D / \tau$ ratios and high damping coefficients (i.e., $\xi>1$ ) fall under this class.

\subsubsection{Model structure 2C (MS-2C)}

Shapes with low $D / \tau$ (first observation) are under second category (MS-2C) as they resemble to the similar relay response of SOPDT systems.

These systems are similar to second order with critically damped behavior, i.e., MS-2C.

$G_{\mathrm{P}}(s)=\frac{K_{\mathrm{P}} \mathrm{e}^{-D s}}{\left(\tau_{\mathrm{P}} s+1\right)^{2}}$

When an unknown process generates relay responses similar to exponentially developed cycles, it can be classified under this category i.e., SOPDT with low $D / \tau$. In fact, most of the systems (including higher order) fall under this class. Similar to model structure, MS-1, MS-2C has three unknown parameters, namely, $K_{\mathrm{P}}, \tau$ and $D$.

\subsubsection{Model structure $2 U$ ( $M S-2 U)$}

The shapes under third observation (lower left corner in Fig. 3) mentioned above, comprise of one or multiple typical under-damped oscillations within one cycle $\left(P_{\mathrm{u}}\right)$. They form a new class or category (MS-2U). Systems under this class has the following model structure

$G_{\mathrm{P}}(s)=\frac{K_{\mathrm{P}} \mathrm{e}^{-D s}}{\left(\tau_{\mathrm{P}}^{2} s^{2}+2 \xi \tau_{\mathrm{P}} s+1\right)}$

where $\xi$ is the damping factor with $\xi<1$.

Both qualitative and quantitative assessments (in latter section) have been presented in this work to confirm these classifications. Nonetheless, these 3 model structures can represent all possible curve shapes generally found in practice.

\section{Identification of transfer functions}

After observing the shapes of the curves of relay responses, a priori guess can be made about the category of the unknown process. The next work is to identify the unknown system parameters for that very model structure (i.e., MS-1, MS-2C, MS-2U). The procedures for identification are explained below (in this work, relay height, $h$ is taken as unity).

\subsection{Model structure 1 (MS-1)}

Responses under this category have sharp edges at peak amplitude and are fully developed at first cycle. Here we discuss about the stable FOPDT systems. The stabilized relay response is modeled analytically [13]. Fig. 4 explains responses obtained from model (thick line, from $t=0$ to $P_{\mathrm{u}} / 2$ as represented by segment $\mathrm{A}-\mathrm{B}$ or $\mathrm{B}-\mathrm{C}$ ) and actual process (thin line) that helps to estimate exact process parameters. The concave

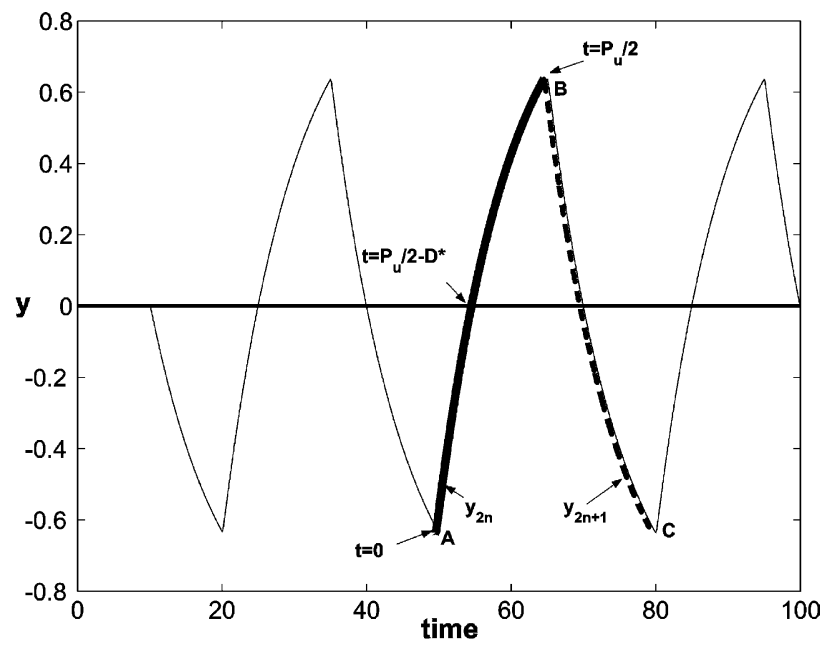

Fig. 4. Relay feedback response for first order plus dead time system (MS-1) and corresponding analytical expression (thick lines) and landmark values. 
upward half period (thick solid line in Fig. 4) can be expressed as

$y(t)=K_{\mathrm{P}} h\left(1-\mathrm{e}^{-t / \tau}\left[\frac{2}{1+\mathrm{e}^{-P_{\mathrm{u}} / 2 \tau}}\right]\right) \quad$ with $t \in\left[0, P_{\mathrm{u}} / 2\right]$

Because this is a strictly monotonic response, observable boundary conditions, giving the landmark points, can be applied directly

$$
\begin{aligned}
& (y)_{t=P_{\mathrm{u}} / 2-D}=0 \\
& (y)_{t=0}=-a \\
& (y)_{t=P_{\mathrm{u}} / 2}=a
\end{aligned}
$$

The effect of time delay, $D$, is inherited in $P_{\mathrm{u}}$ as it is evident in Eq. (4) above. The time delay, $D$, is the time taken to reach peak of relay response $[17,10,16]$.

The above equation can be solved for $K_{\mathrm{P}}$ and $\tau$ with the help of above boundary conditions. Using first boundary condition (Eq. (5)) to Eq. (4) one can find time constant, $\tau$, iteratively from following equation

$2 \mathrm{e}^{\left(D-P_{\mathrm{u}} / 2\right) / \tau}-\mathrm{e}^{-P_{\mathrm{u}} / 2 \tau}-1=0$

Substitution of second initial condition (Eq. (6)) in Eq. (4) leads to

$$
K_{\mathrm{P}}=\frac{-a}{h\left(1-\left[2 /\left\{1+\mathrm{e}^{-P_{\mathrm{u}} / 2 \tau}\right\}\right]\right)}
$$

from which process gain, $K_{\mathrm{P}}$, can be found out.

Therefore identification consists of following steps:

(0) Measure the time taken to reach peak amplitude $(D)$, peak amplitude $(a)$ and period of oscillation $\left(P_{\mathrm{u}}\right)$ from stabilized part of response.

(1) Compute time constant, $\tau$, from Eq. (5). For the purpose of iteration, initial guess value of $\tau$ can be taken as

$$
\tau=\frac{\tan \left(\pi-D \omega_{\mathrm{u}}\right)}{\omega_{\mathrm{u}}}
$$

(2) Compute $K_{\mathrm{P}}$ from Eq. (6) above.

\subsection{Model structure $2 C(M S-2 C)$}

Systems under this category has three unknown parameters, namely, process gain, $K_{\mathrm{P}}$, process time constant, $\tau$ and time delay, $D$. The analytical expression for the stabilized relay response is given by Panda and $\mathrm{Yu}$ [13] (Fig. 5).

$$
\begin{aligned}
y(t)= & K_{\mathrm{P}} h\left\{1-\mathrm{e}^{-\frac{t}{\tau}}\left[\frac{2}{1+\mathrm{e}^{-P_{\mathrm{u}} / 2 \tau}}\right]\right. \\
& \left.-2 \mathrm{e}^{-\frac{t}{\tau}}\left[\frac{t / \tau}{1+\mathrm{e}^{-P_{\mathrm{u}} / 2 \tau}}+\frac{-\left(P_{\mathrm{u}} / 2 \tau\right) \mathrm{e}^{-P_{\mathrm{u}} / 2 \tau}}{\left(1+\mathrm{e}^{-P_{\mathrm{u}} / 2 \tau}\right)^{2}}\right]\right\} \\
t \in & {\left[0, P_{\mathrm{u}} / 2\right] }
\end{aligned}
$$

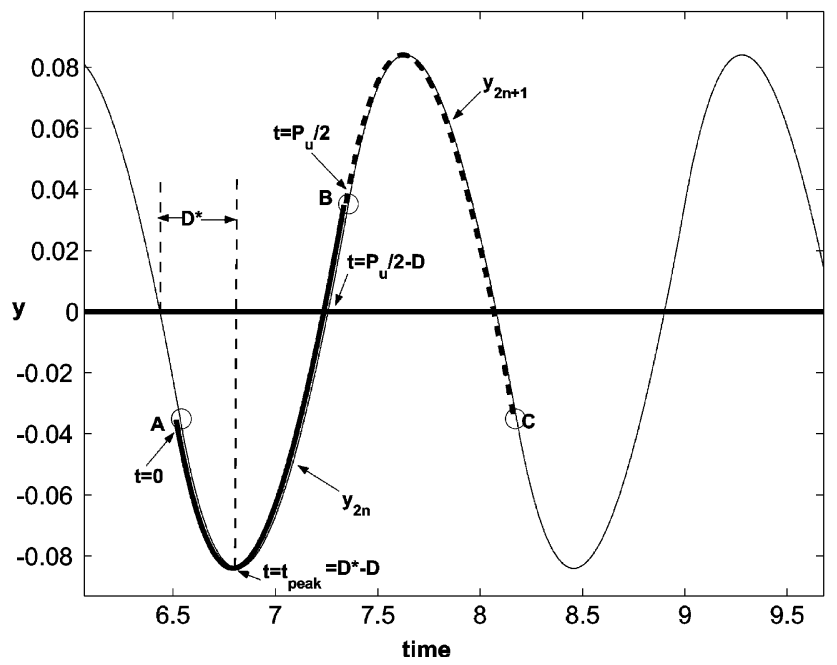

Fig. 5. Relay feedback response for second order critically damped system (MS-2C) and corresponding analytical expression (thick lines) and landmark values.

with boundary conditions as follows:

$$
\begin{aligned}
& (y)_{t=t_{\text {peak }}}= \pm a, \\
& (y)_{t=P_{\mathrm{u}} / 2-D}=0 \\
& \left(\frac{\mathrm{d} y}{\mathrm{~d} t}\right)_{t=t_{\text {peak }}}=0
\end{aligned}
$$

As in this case, relay response (Fig. 5) shows nonmonotonous behavior, initial point of the response is unknown. Hence, time delay $(D)$ can not be directly measured, as previously, as time taken to reach the peak of response. One observable parameter, is the apparent dead time $D^{*}$ which is defined as the time to reach peak of relay response produced by unknown system. Moreover, observable boundary conditions (landmark points) cannot be applied directly, because the starting point of the RFB response of analytical expression for half period described by Eq. (8) is unknown. Therefore additional parameters need to be introduced from boundary conditions mentioned in Eqs. (12)-(14). It can be noticed from Fig. 5 that,

$t_{\text {peak }}=D^{*}-D$

Substitution of boundary condition Eq. (14) into Eq. (11) gives rise to

$D^{*}-D=\frac{P_{\mathrm{u}}}{2} \frac{1}{1+\mathrm{e}^{P_{\mathrm{u}} / 2 \tau}}$

Substitution of Eq. (13) into Eq. (11) leads to

$$
\left(1+\mathrm{e}^{P_{\mathrm{u}} / 2 \tau}\right)^{2}=\frac{2 D \mathrm{e}^{D / \tau}}{\tau}\left[\left(\frac{P_{\mathrm{u}}}{2 D}-1\right) \mathrm{e}^{P_{\mathrm{u}} / 2 \tau}-1\right]
$$

Hence, the following steps are followed to estimate the unknown model parameters: 
(0) Read apparent dead time $\left(D^{*}\right)$, i.e., time taken by actual relay response to reach its peak, amplitude $(a)$ and ultimate period $\left(P_{\mathrm{u}}\right)$ from relay feedback response data.

(1) Solve simultaneously Eqs. (15) and (16) to find out $D$ and $\tau$.

(2) Calculate $K_{\mathrm{P}}$ from following relation:

$K_{\mathrm{P}}\left\{1-y_{1} \mathrm{e}^{-t_{\text {peak }} / \tau}-\mathrm{e}^{-t_{\mathrm{P}} / \tau}\left(y_{1} \frac{t_{\text {peak }}}{\tau}-\frac{P_{\mathrm{u}}}{4 \tau} y_{1}^{2} \mathrm{e}^{-0.5 P_{\mathrm{u}} / \tau}\right)\right\}=a$

where $t_{\text {peak }}=D^{*}-D \quad$ and $\quad y_{1}=\frac{2}{1+\mathrm{e}^{-0.5 P_{\mathrm{u}} / \tau}}$

\subsection{Model structure $2 U(M S-2 U)$}

SOPDT systems with low damping coefficient, $\xi$, and moderate to high $D / \tau$ ratio fall under this category. This has been verified by multiplicative error analysis (presented in later section). This type of processes has four unknown parameters, namely, $K_{\mathrm{P}}, \tau, \xi$ and $D$, as it appears in Eq. (3). The analytical expression for the relay feedback response of this kind of processes is given as [13].

$y(t)=K_{\mathrm{P}} h\left\{1-2 \frac{\mathrm{e}^{\frac{-\xi t}{\tau}}}{\beta} \sin \left(\frac{\beta t}{\tau}+\alpha\right)\right\}$

here $\alpha=\tan ^{-1}\left(\frac{\beta+\beta r \cos (\theta)-\xi r \sin (\theta)}{\xi+\xi r \cos (\theta)+\beta r \sin (\theta)}\right)$

where $r=\mathrm{e}^{-\frac{P_{\mathrm{u}} \xi_{\tau}}{2}}$ and $\theta=\frac{P_{\mathrm{u}} \beta \tau}{2}$ and $\beta=\sqrt{1-\xi^{2}}$

This Eq. (19) with following boundary conditions can be used to estimate above mentioned four unknown parameters

$(y)_{t=P_{\mathrm{u}} / 2}=a$

$\left(\frac{\mathrm{d} y}{\mathrm{~d} t}\right)_{t=t_{\text {peak }}}=0$

$(y)_{t=0}+(y)_{t=P_{\mathrm{u}} / 2}=0$

$(y)_{t=P_{\mathrm{u}} / 2-D}=0$

Here we find two different scenarios in the left part of Fig. 3. In the upper portion of this part, the response is smooth and having a single peak in each cycle whereas in the lower portion (left corner), the response has more than one peak per cycle (easy to locate landmark points). The landmarks are starting point $(t=0)$, ending point $\left(t=P_{\mathrm{u}} / 2\right), t_{\text {peak }}$ and zero-crossing of response. If

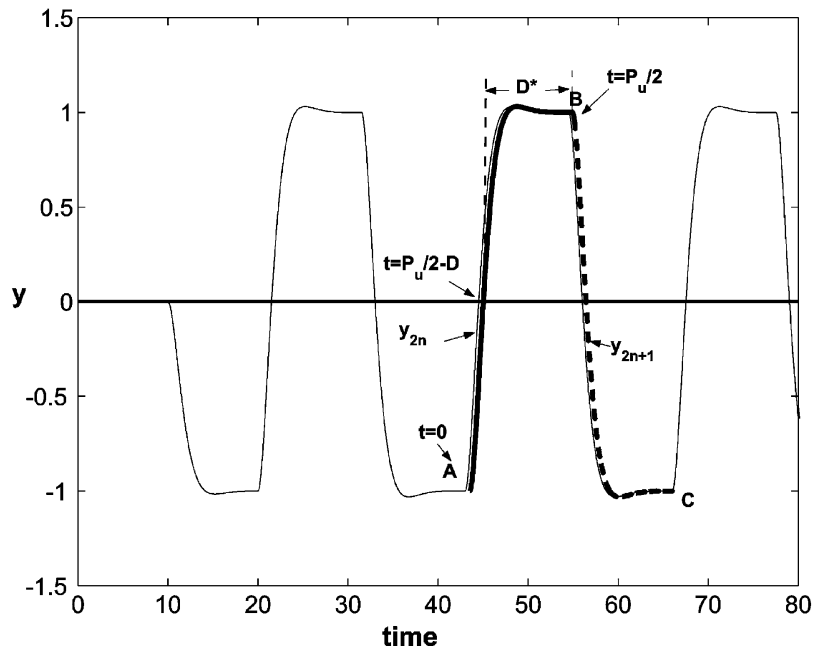

Fig. 6. Relay feedback response for second order underdamped system (MS-2U) large $D / \tau(>1)$ and corresponding analytical expression (thick lines) and landmark values.

one can locate point A or B (as in Fig. 6) on the relay response obtained from experiment, then it becomes easy to find equations to calculate unknown parameters. But the real problem is to find out starting and ending points of response. We assume the following to formulate the Eqs. (21)-(24).

(i) The relay responses are symmetric about zero base line.

(ii) The response of processes that shows more than one peak per cycle (second scenario) starts from point $\mathrm{A}$ and ends at point $\mathrm{B}$ (as shown in Fig. 6).

(iii) For the first scenario, the response starts from bottom most point and ends at next top point (as in Fig. 7).

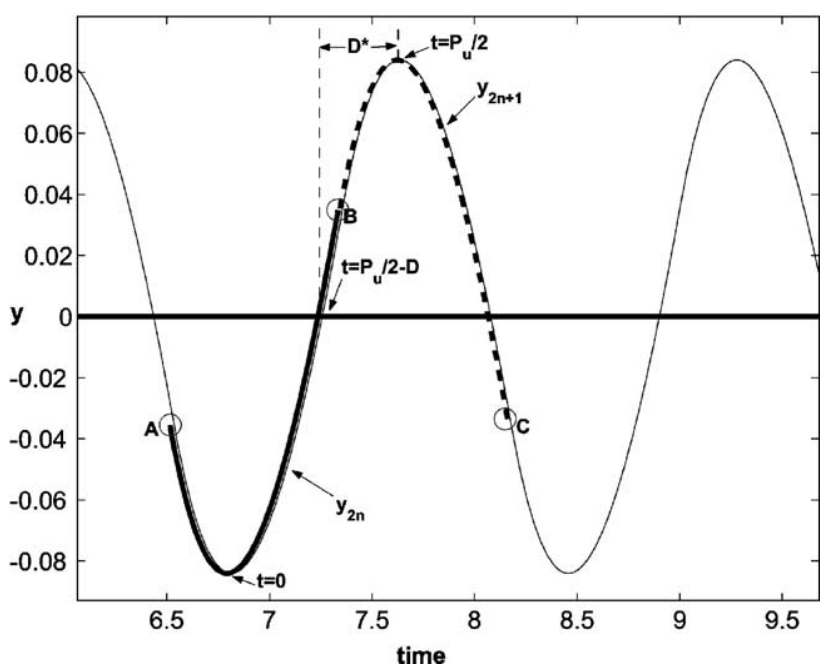

Fig. 7. Relay feedback response for second order underdamped system (MS-2U) with small $D / \tau(<1)$ and corresponding analytical expression (thick lines) and landmark values. 
(iv) If more than one peak exists, we consider the last peak for calculation of $D^{*}$ ).

Now $t_{\text {peak }}$ can be written as $t_{\text {peak }}=D^{*}-D+P_{\mathrm{u}} / 2$. Substituting Eq. (21) in Eq. (19), we get

$K_{\mathrm{P}}\left\{1-\frac{2}{\beta} \mathrm{e}^{-\xi P_{\mathrm{u}} / 2 \tau}\left[\sin \left(\frac{\beta P_{\mathrm{u}}}{2 \tau}+\alpha\right)\right]\right\}=a$

condition in Eq. (22) gives

$\frac{\tau}{\beta}\left[\pi+\cos ^{-1} \xi-\alpha\right]-t_{\text {Peak }}=0$

Replacing Eq. (23) in Eq. (19) yields

$\frac{1}{\beta}\left[\sin (\alpha)+\mathrm{e}^{-\xi P_{\mathrm{u}} / 2 \tau} \sin \left(\frac{\beta P_{\mathrm{u}}}{2 \tau}+\alpha\right)\right]=1$

and according to Eq. (24) one can obtain from Eq. (19)

$K_{\mathrm{P}}\left\{1-\frac{2}{\beta} \mathrm{e}^{-\xi\left(0.5 P_{\mathrm{u}}-D\right) / \tau}\left[\sin \left(\frac{\beta\left(0.5 P_{\mathrm{u}}-D\right)}{\tau}+\alpha\right)\right]\right\}=0$

These equations can be solved simultaneously to find $K_{\mathrm{P}}, D, \tau$ and $\xi$.

Alternatively, a simple procedure can be adopted. The dead time $(D)$ and time needed to reach peak $\left(t_{\text {peak }}\right)$ can be easily measured from relay response curve (see Fig. 6). Time from point $t=0.5 P_{\mathrm{u}}-D$ to point $\mathrm{B}$ gives a measure of dead time $D$. Height of point $\mathrm{B}$ (where $t=0.5 P_{\mathrm{u}}$ ) from zero base line gives an estimate of $K_{\mathrm{P}}$. Other two unknown parameters ( $\tau$ and $\xi$ ) can be found separately from the above Eqs. (25) and (26).

Hence the following steps may be followed to estimate the four unknown parameters of MS-2U systems:

(0) Estimate apparent dead time $\left(D^{*}\right)$, i.e., time taken by actual relay response to reach its second peak. In case, there is more than one peak in a particular cycle of RFB response, we consider the last peak of that cycle for estimation of $D^{*}$.

(1) Solve Eqs. (25)-(28) simultaneously to calculate $K_{\mathrm{P}}, \tau, \xi$ and $D$.

This procedure enables us to find parameters for MS$2 \mathrm{U}$ type of models.

\section{Validation of model structures}

All the (unknown) process models (63) under study are identified separately as MS-1, MS-2C, and MS-2U using aforementioned algorithms. Thus each of the process model will have three model structures. Multiplicative error (described in latter section) in frequency domain (IAE) is calculated (with respect to true process)

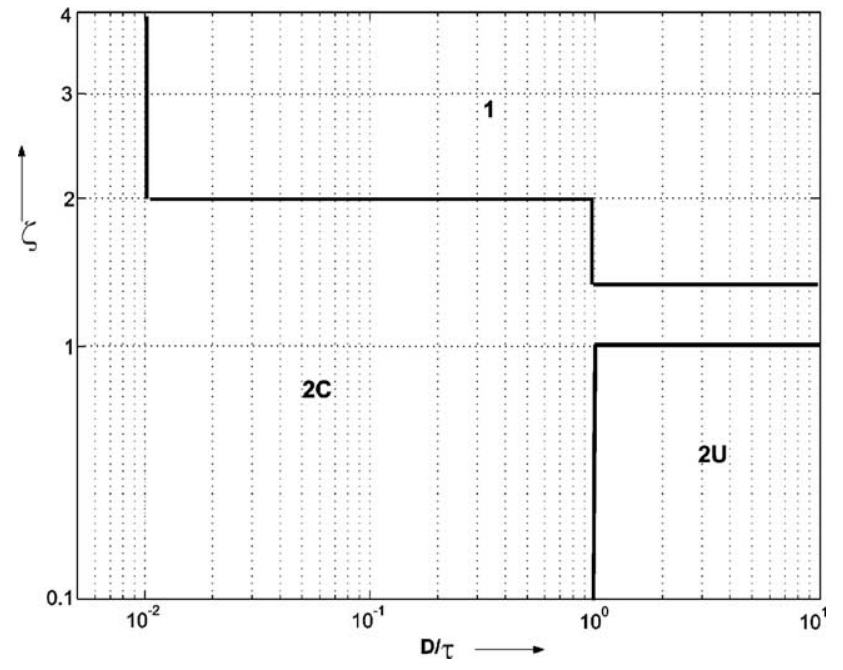

Fig. 8. Quantitative classification of different model structures based on frequency domain IAE values.

for each model structures of each (unknown) process. A minimum IAE was sort (out of three IAE thus obtained) for each (unknown) process to confirm the exact class in which the process belongs. The classification thus obtained is shown in Fig. 8. Processes with $D / \tau \leqslant 1$ fall under $2 \mathrm{C}$ class. MS-2C also covers different processes with $\xi=1.0$. Systems with $D / \tau>1$ and $\xi<1$ fall under MS-2U. Several processes with $D / \tau>1$ and $\xi>1$ constitute the region for MS-1 type of system. Thus this figure shows that MS-2C covers largest parameter space for the systems studied. MS-1 followed by MS-2U accordingly covers rest of the portion of parameter space $(\xi$ vs $D / \tau)$.

The validity of the above identification method is found by selecting six examples arbitrarily having shapes of RFB responses with different characteristics. Relay feedback tests are performed on these examples and are shown in Fig. 9 as "TRUE". Ultimate properties $\left(K_{\mathrm{u}}\right.$ and $\left.P_{\mathrm{u}}\right)$ of these unknown processes (examples) are tabulated in Table 1. After identifying these examples as different possible model structures (using the identification procedures described above), (1) time domain relay feedback response are reproduced and (2) frequency domain multiplicative error are calculated to compare with that of TRUE.

The equivalent models of six examples, after identifying them as MS-1, MS-2C and MS-2U, are presented in Table 1. Each equivalent model of one example has same values of $K_{\mathrm{u}}$ and $\omega_{\mathrm{u}}$ but has different structures. Relay feedback tests are conducted on these model structures (MS-1, MS-2C and MS-2U) and are shown in Fig. 9. Comparing the shapes of RFB responses of these possible three model structures of one example, one by one, with that of its TRUE RFB response, one can find out the correct model structure of the example. 


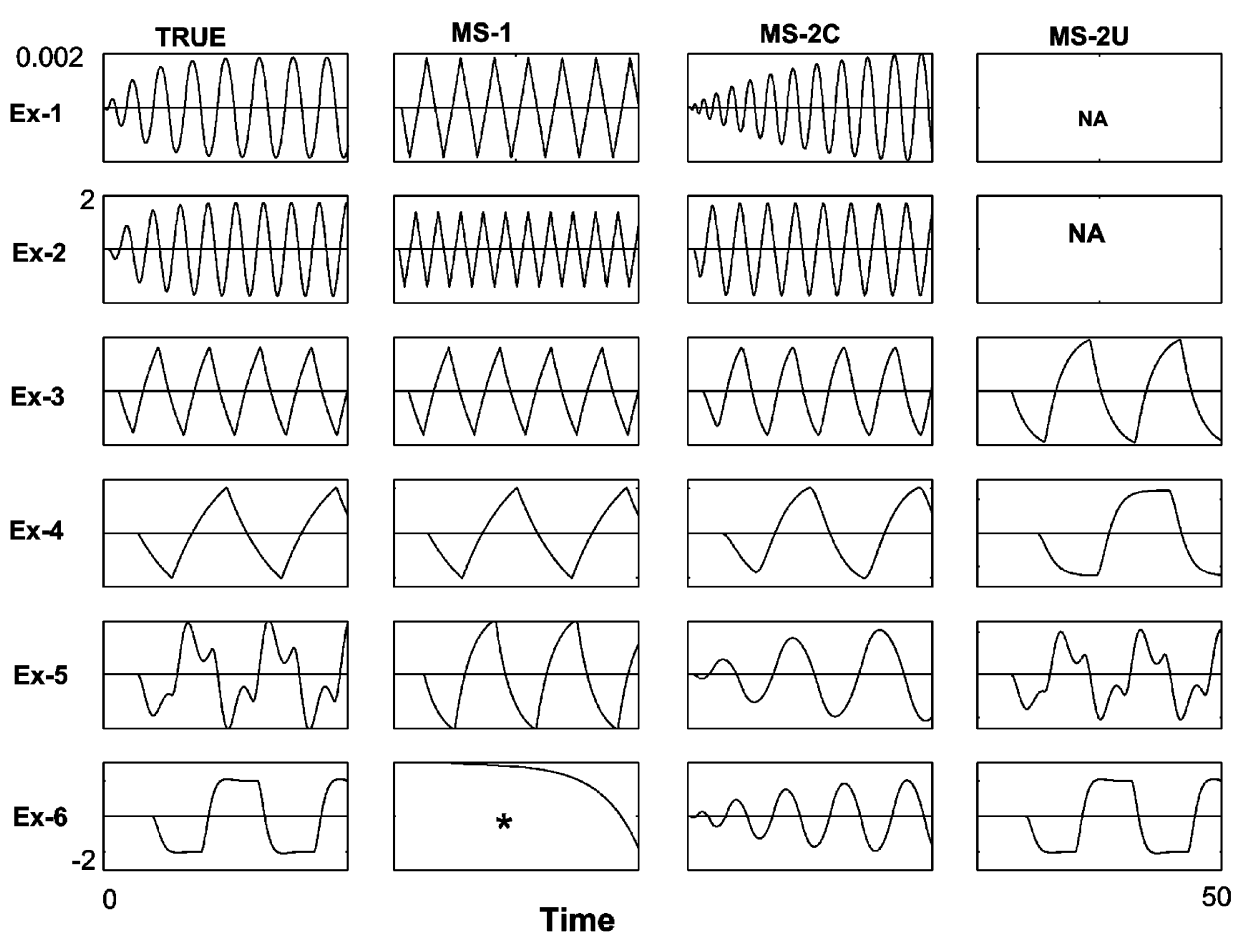

Fig. 9. Time domain relay feedback responses with six examples $\left({ }^{*}\right.$ indicates limit cycle not possible, and NA denotes not applicable).

Table 1

True process with their ultimate properties and identified models under different model structure

\begin{tabular}{|c|c|c|c|c|c|c|}
\hline Example & TRUE process & $K_{\mathrm{u}}$ (true proc.) & $P_{\mathrm{u}}$ (true proc.) & $\mathrm{MS}-1^{\mathrm{a}}$ & MS- $2 C^{b}$ & $\mathrm{MS}-2 \mathrm{U}^{\mathrm{c}}$ \\
\hline 1 & $\frac{1.0 \mathrm{e}^{-0.01 s}}{s^{2}+6.0 s+1}$ & 544.4977 & 0.277 & $\frac{-0.0249 \mathrm{e}^{-0.066 s}}{-0.7367 s+1}$ & $\frac{1.106 \mathrm{e}^{-0.0022 s}}{(1.066 s+1)^{2}}$ & $\frac{0.0027 \mathrm{e}^{-0.1108 s}}{3.5 E-4 s^{2}+0.03 s+1}$ \\
\hline 2 & $\frac{1.0 \mathrm{e}^{-1.0 s}}{s^{2}+0.4 s+1}$ & 0.48726 & 6.68 & $\frac{-206.1472 \mathrm{e}^{-1.41 s}}{-112.0192 s+1}$ & $\frac{5.3954 \mathrm{e}^{-1.1566 s}}{(1.2239 s+1)^{2}}$ & $\frac{2.6129 \mathrm{e}^{-2.6995 s}}{0.0077 s^{2}+0.17 s+1}$ \\
\hline 3 & $\frac{1.0 \mathrm{e}^{-3.0 s}}{s^{2}+6 s+1}$ & 3.1419 & 10.435 & $\frac{1.1901 \mathrm{e}^{-3.062 s}}{7.3552 s+1}$ & $\frac{0.5302 \mathrm{e}^{-2.9187 s}}{(1.4636 s+1)^{2}}$ & $\frac{2.2016 \mathrm{e}^{-6.8515 s}}{0.816 s^{2}+3.64 s+1}$ \\
\hline 4 & $\frac{1.0 \mathrm{e}^{-7.0 s}}{s^{2}+10 s+1}$ & 2.1591 & 21.613 & $\frac{1.036 \mathrm{e}^{-7.03 s}}{10.4411 s+1}$ & $\frac{0.5984 \mathrm{e}^{-6.8595 s}}{(2.6851 s+1)^{2}}$ & $\frac{0.6348 \mathrm{e}^{-12.2007 s}}{2.377 s^{2}+3.08 s+1}$ \\
\hline 5 & $\frac{1.0 \mathrm{e}^{-7.0 s}}{s^{2}+0.4 s+1}$ & 0.61948 & 16.655 & $\frac{2.08 \mathrm{e}^{-7.1935 s}}{-1.65 s+1}$ & $\frac{60.5766 \mathrm{e}^{1.025 s}}{(15.9654 s+1)^{2}}$ & $\frac{1.05 \mathrm{e}^{-6.8902 s}}{1.03 s^{2}+0.41 s+1}$ \\
\hline 6 & $\frac{1.0 \mathrm{e}^{-10.0 s}}{s^{2}+1.6 s+1}$ & 1.2358 & 23.009 & $\frac{-1.59 \mathrm{e}^{-10.0 s}}{-7.49 s+1}$ & $\frac{82.1116 \mathrm{e}^{-2.2419 s}}{(24.4624 s+1)^{2}}$ & $\frac{1.0 \mathrm{e}^{-10.0 s}}{s^{2}+1.6 s+1}$ \\
\hline
\end{tabular}

${ }^{\text {a }}$ First order plus dead time.

${ }^{\mathrm{b}}$ Second order critically damped plus dead time.

${ }^{c}$ Second order underdamped plus dead time.

Thus it is confirmed that examples 1 and 2 (Ex-1, Ex-2) belong to MS-2C, examples 3 and 4 belong to MS- 1 and example 5 and 6 belong to MS-2U. The results clearly indicate the strength of the identification algorithm to extract the correct model structure.

Identification of unknown systems (six examples) under right category/model structure is validated by calculating the integrated absolute error (IAE) of each of these equivalent models. IAE is evaluated as
Table 2

Values of integral of absolute IAE in frequency range of $0.1 \omega_{\mathrm{u}}$ to $10 \omega_{\mathrm{u}}$

\begin{tabular}{lllll}
\hline Example & MS-1 & MS-2C & MS-2U & Recommended category \\
\hline 1 & 0.2104 & 0.0033 & 0.3683 & MS-2C \\
2 & 0.3261 & 0.0559 & 0.2864 & MS-2C \\
3 & 0.0236 & 0.1156 & 2.2728 & MS-1 \\
4 & 0.0121 & 0.1274 & 1.3599 & MS-1 \\
5 & 0.5990 & 0.6196 & 0.0662 & MS-2U \\
6 & 1.2370 & 0.4837 & 0 & MS-2U \\
\hline
\end{tabular}



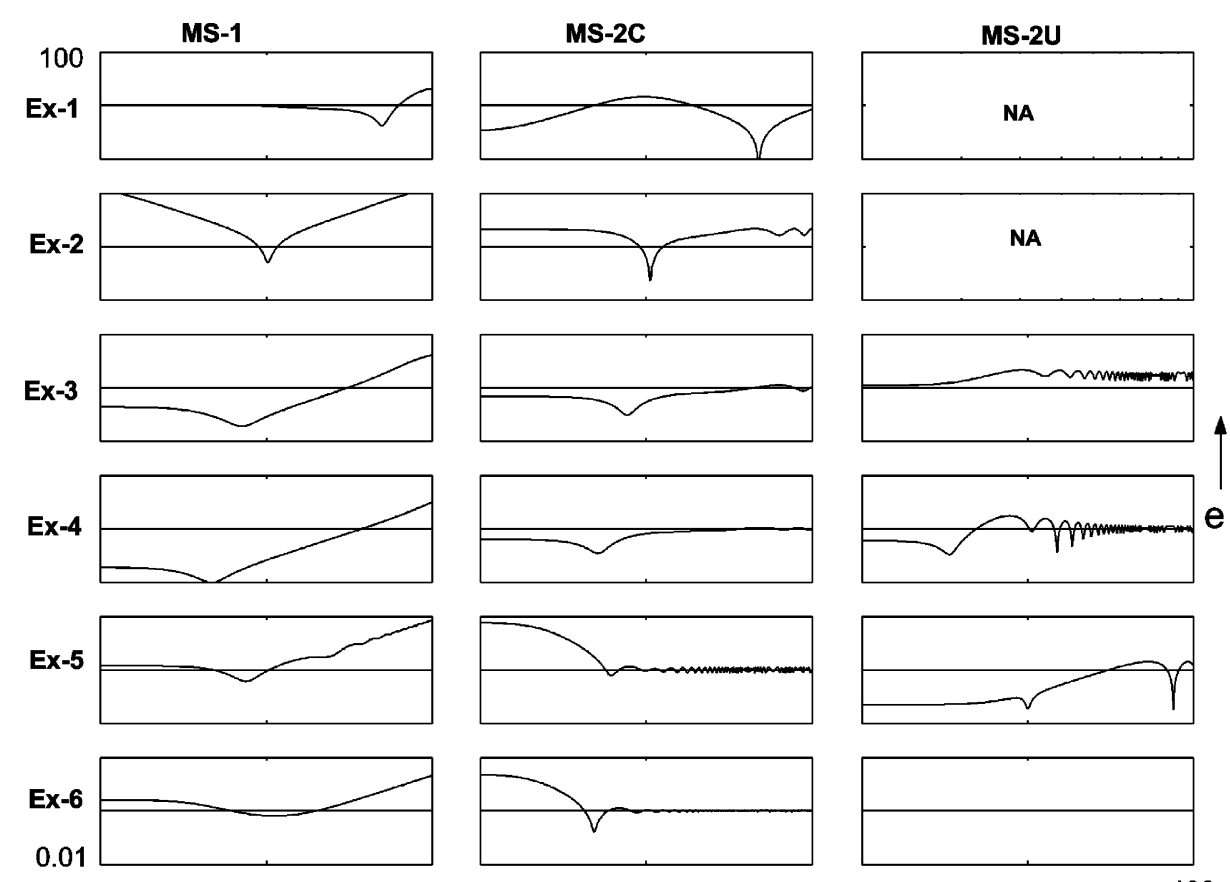

$\omega$
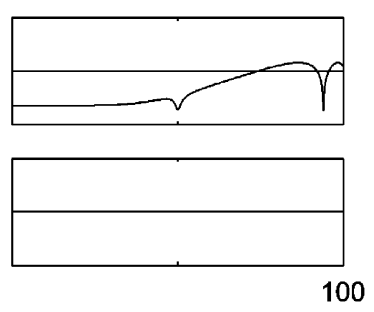

Fig. 10. Frequency domain multiplicative error of six examples (NA denotes not applicable).

$I A E_{\omega}=\int_{0.1 \omega_{\mathrm{u}}}^{10 \omega_{\mathrm{u}}}\left|\frac{G_{\mathrm{m}}(j \omega)-G_{\mathrm{P}}(j \omega)}{G_{\mathrm{P}}(j \omega)}\right| \mathrm{d} \omega$

where $G_{\mathrm{P}}$ is the true process and $G_{\mathrm{m}}$ is the identified model.

Eq. (25) is a measure of frequency domain multiplicative error between TRUE process and a particular model structure of one example. Table 2 shows quantitative IAE values of frequency domain error. Out of three model structures for a particular example, one yields lowest IAE and falls under that category. The results clearly indicate that examples 1 and 2 belong to MS-2C, example 3 and 4 fall under category 1 whereas example 5 and 6 belong to MS-2U. Fig. 10 show the frequency domain multiplicative error plots for the examples undertaken. Thus frequency domain IAE values are helpful to compare and find out exact model structure for an unknown process.

\section{Disturbance and noises}

\subsection{Load disturbance}

Load changes frequently occur in process industries. Disturbance rejection is a major criterion in chemical process control. Sensitivity with respect to load changes is an important consideration in evaluating identification techniques.

Under load disturbance ( $L=0.5$ to a process model with transfer functions as given in Table 3), an ideal relay feed back test results in an asymmetric oscillation (Fig. 11), and consequently, an imbalance in half periods results that leads to errors in estimates of $K_{\mathrm{u}}$ and $\omega_{\mathrm{u}}$. To overcome this load effect, a bias value $\left(\delta_{0}\right)$ is added to the relay-input-height $(h)$. Yu [18] has shown that this bias value is related to amplitude of oscillation by following relation:

Table 3

Details of processes for the study of disturbance rejection

\begin{tabular}{lllllllll}
\hline Model structures & $\begin{array}{l}\text { True process } \\
\text { transfer function }\end{array}$ & $\left(K_{\mathrm{u}}\right)_{\mathrm{b}}$ & $\left(K_{\mathrm{u}}\right)_{\mathrm{u}}$ & $\left(K_{\mathrm{u}}\right)_{\mathrm{a}}$ & $\left(\omega_{\mathrm{u}}\right)_{\mathrm{b}}$ & $\left(\omega_{\mathrm{u}}\right)_{\mathrm{u}}$ & $\left(\omega_{\mathrm{u}}\right)_{a}$ & Remarks \\
\hline MS-1 & $\frac{1.0 \mathrm{e}^{-10 s}}{s^{2}+10 s+1}$ & 2.0013 & 4.1901 & 2.0013 & 0.2095 & 0.1920 & 0.2095 & Shapes can be restored \\
MS-2C & $\frac{1.0 \mathrm{e}^{-1 s}}{s^{2}+2 s+1}$ & 2.5543 & 2.5508 & 2.5542 & 1.3159 & 1.2955 & 1.3157 & Shapes can be restored \\
MS-2U & $\frac{1.0 \mathrm{e}^{-10 s}}{s^{2}+1.2 s+1}$ & 1.0702 & 0.5000 & 1.0702 & 0.2765 & 0.2759 & 0.2765 & Shapes can be restored \\
\hline
\end{tabular}

b: before load disturbance, a: after adjusting h, u: under load disturbance. 

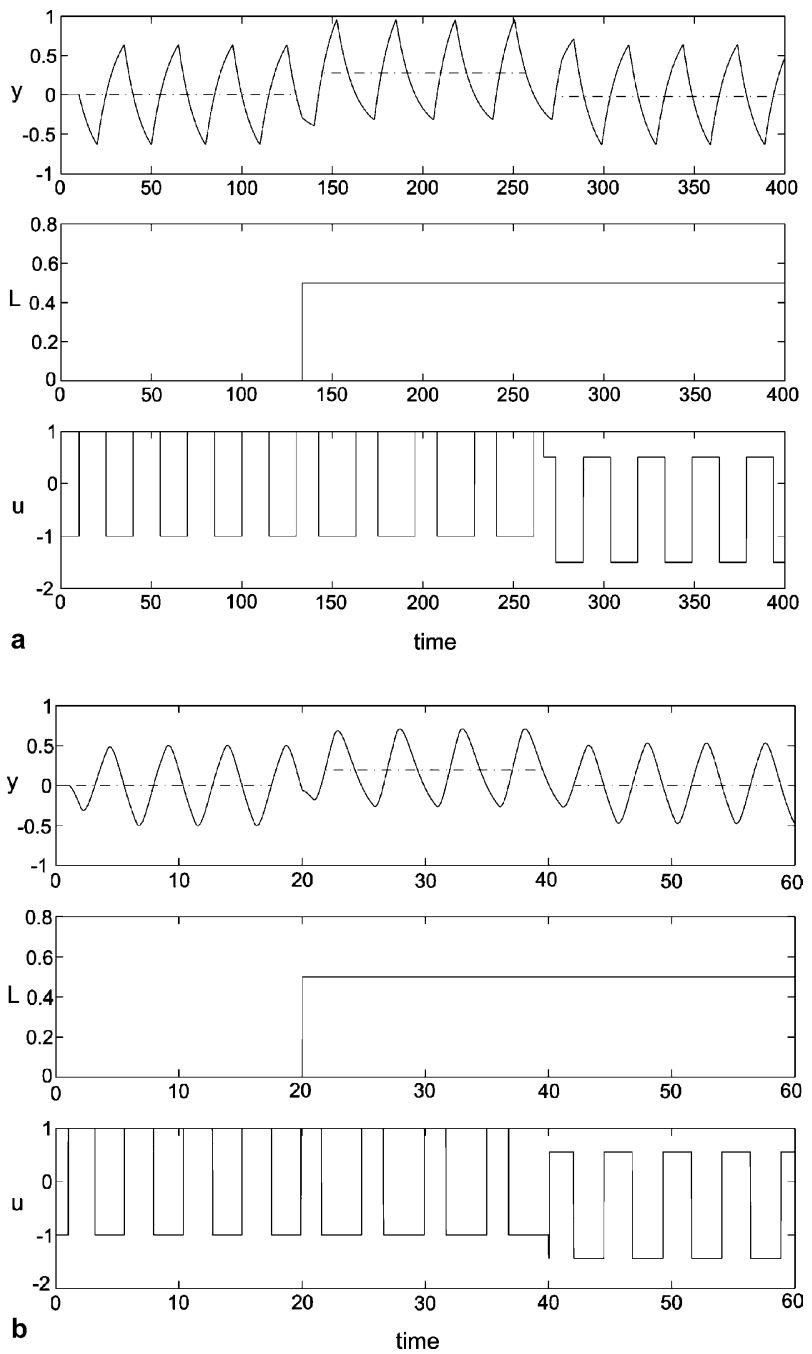

Fig. 11. Use of output biased relay to restore the correct shape under load disturbance for (a) MS-1 and (b) MS-2C systems (transfer functions and limit cycle data of the processes are given in Table 3).

$\delta_{0}=-\left(\frac{\Delta a}{a}\right) h$

where $\Delta a$ is asymmetry in the output and $a$ is the amplitude of output relay response. Thus to restore the symmetry in output response, the relay is switched to an output bias so that the newly adjusted relay input height becomes $H=h+\delta_{0}$.

Let us take an example of MS-2C with transfer function, $G=\frac{1.0 \mathrm{e}^{-1 s}}{(s+1)^{2}}$ as mentioned in Table 3. Without load disturbance, the relay feedback test (with $h=0.8$ ) gives $K_{\mathrm{u}}=2.5543$ and $\omega_{\mathrm{u}}=1.3159$ and the response is shown in Fig. 11(a) $(t=0-20)$. After introducing a load change of $L=0.02$ at $t=20$, an asymmetric sustained oscillation results $(t=20-40)$. The estimated values of $K_{\mathrm{u}}$ and $\omega_{\mathrm{u}}$ become 2.5508 and 1.2955 respectively. From the system response we have $\Delta a=-0.16$ and $a=0.4$. With the known values of $\Delta a$ and $a$, the bias value $\left(\delta_{0}\right)$ can be computed and the result becomes $\delta_{0}=0.32$. Next, an output biased relay feedback test is performed $(t>40)$ (with the same load still active) and $K_{\mathrm{u}}$ and $\omega_{\mathrm{u}}$ are found to be 2.5542 and 1.3157 respectively, which are almost same that of disturbance free case. Similar tests have been performed with other examples ( $h=1$ and $L=0.5$ was given) and significant parameter values are shown in Table 3 . In each case, the shapes, $K_{\mathrm{u}}$ and $\omega_{u}$ values were regained. Fig. 11 shows that the asymmetry in sustained oscillation induced by load disturbance is removed by output biased relay. Therefore, output biased relay is very effective in maintaining the quality of the model in the face of load changes.

\subsection{Presence of measurement noise}

Measurement noise is a common problem in almost all process industries. It is necessary to know whether the shapes of relay feedback responses are deteriorated in the presence of noise or not. The proposed method for model identification was tested against measurement noise. Two different processes (one belongs to MS-2C and the other belongs to MS-2U) are undertaken for study. They are

$G=\frac{1.0 \mathrm{e}^{-0.1 S}}{(s+1)^{2}}$

$G=\frac{1.0 \mathrm{e}^{-10 s}}{s^{2}+1.6 s+1}$

Relay feedback tests (relay height $=1$ ) were performed on these processes with noise. Noise to signal ratio (NSR) was 0 and $1 / 5$. The relay feedback responses are shown in Fig. 12. In case of second process (Eq. (32)) hysteresis was used to switch the relay input at $\varepsilon=0.2$ to restore the shape at initial stage of response. The limit cycle data were calculated by taking average of fictitious peaks around the nominal peak of the stabilized response. Thus amplitude $\left(a^{\prime}\right)$ and period of oscillation $\left(P_{\mathrm{u}}^{\prime}\right)$ were found. The above mentioned identification algorithms were used to evaluate the model structures and are shown in Table 4. It can be seen that the calculated limit cycle data from noisy RFB response curves are almost closure to that of noise-free case. Hence identified model parameters are in close approximation to the process model.

\section{Controller tuning and performance}

After identifying the model structure of the unknown process, it is necessary to find a proper controller and its tuning rule for the process. Choice of a suitable controller and its tuning are discussed in the following section. 

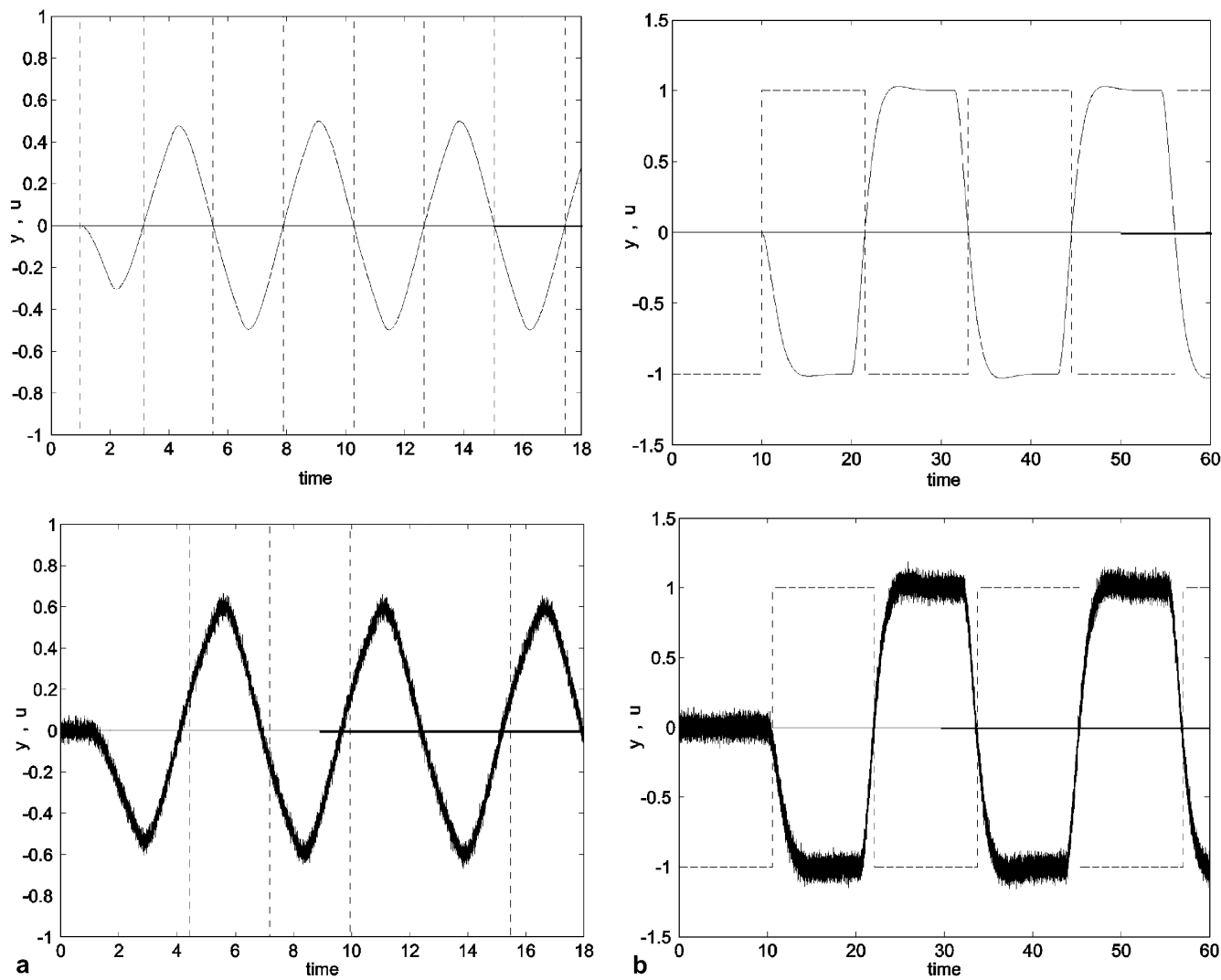

Fig. 12. Relay feedback responses for processes (A) (example 1 in Table 4) and (B) (example 2 in Table 4) without and with measurement noise $(\mathrm{NSR}=0.2)$.

Table 4

Detail of process identification in presence of measurement noise

\begin{tabular}{|c|c|c|c|c|}
\hline True process & \multicolumn{2}{|c|}{ Identified process with NSR $=0$} & \multicolumn{2}{|c|}{ Identified process with NSR $=1 / 5$} \\
\hline $1.0 \mathrm{e}^{-1.0 s}$ & $0.9946 \mathrm{e}^{-1.0029 s}$ & $a=0.4984 P_{\mathrm{u}}=4.775$ & $1.0129 \mathrm{e}^{-1.0357 s}$ & $a^{\prime}=0.4985 P_{u}^{\prime}=4.988$ \\
\hline$\overline{(s+1)^{2}}$ & $(0.996 s+1)^{2}$ & & $(1.0559 s+1)^{2}$ & \\
\hline$\frac{1.0 \mathrm{e}^{-10.0 s}}{s^{2}+1.6 s+1}$ & $\frac{1.0 \mathrm{e}^{-10.0 s}}{s^{2}+1.6 s+1}$ & $a=1.0303 P_{\mathrm{u}}=23.009$ & $\frac{1.0111 \mathrm{e}^{-9.9476 s}}{0.984 s^{2}+1.63 s+1}$ & $a^{\prime}=1.03186 P_{\mathrm{u}}^{\prime}=22.92$ \\
\hline
\end{tabular}

\subsection{Model structure 1}

FOPDT systems with low $D / \tau$ value are better controlled by PI controller and appropriate tuning rules are Tyreus-Luyben (TL) or IMC as discussed by Thyagarajan and $\mathrm{Yu}$ [16]. PID controller (with IMC tuning) may be suggested for MS-1 type of processes with large $D / \tau$ ratio [11]. PID with IMC-Maclaurian tuning can also be used for these systems.

\subsection{Model structure $2 C$}

It is well known that PID controller is applicable for second order plus dead time systems. In a separate attempt [14] and from our experience in the choice/selec- tion of tuning rules of PID controller for SOPDT processes with wide range of $D / \tau$ and $\xi$ values and it is concluded that processes with $D / \tau \leqslant 0.1$ require Tyreus-Luyben tuning, processes with $0.1<D / \tau \leqslant 1$ need IMC-Chien [4], whereas processes with $D / \tau>1$ are recommended to use IMC-Mac [6] for better performance. Table 5 shows detail of selection and applicability of proper tuning rules for PID controllers. Fig. 13 helps in selecting appropriate PID tuning rules for particular category or model structure.

\subsection{Model structure $2 U$}

Tuning rules like Honeywell tuning, IMC types of tuning and tuning rules based on direct synthesis 
Table 5

PI/PID tuning rules for different model structures

\begin{tabular}{|c|c|c|c|c|}
\hline Model & Parameter & Controller & Tuning rules & Tuning formula \\
\hline \multirow[t]{7}{*}{ MS-1 } & $(D / \tau<0.1)$ & PI & Tyreus-Luyben & $K_{C}=\frac{K_{\mathrm{u}}}{3.2}$ and $\tau_{I}=2.2 P_{\mathrm{u}}$ \\
\hline & $(0.1 \leqslant D / \tau \leqslant 1)$ & PI & Min ITAE (Rovira) & $K_{C}=\frac{0.586}{K_{\mathrm{P}}}\left(\frac{\tau}{D}\right)^{0.916}$ and $\tau_{I}=\frac{\tau}{1.03-0.165\left(\frac{D}{\tau}\right)}$ \\
\hline & $(D / \tau>1)$ & PI & IMC & $K_{C}=\frac{\tau+D / 2}{K_{\mathrm{P}} \lambda}$ and $\tau_{I}=\tau+D / 2$ where $\lambda=\max (1.7 D, 0.2 t)$ \\
\hline & $(D / \tau<0.1)$ & PID & Tyreus-Luyben & $K_{C}=\frac{K_{\mathrm{u}}}{2.2}, \tau_{I}=2.2 P_{\mathrm{u}}$ and $\tau_{D}=\frac{P_{\mathrm{u}}}{6.3}$ \\
\hline & $(0.1 \leqslant D / \tau<1)$ & PID & IMC-Chien & $K_{C}=\frac{\tau}{K_{\mathrm{P}}(\lambda+D)}, \tau_{I}=2 \tau$ and $\tau_{\mathrm{D}}=\tau / 2$ \\
\hline & \multirow[t]{2}{*}{$(D / \tau \geqslant 1)$} & \multirow[t]{2}{*}{ PID } & \multirow[t]{2}{*}{ IMC-MAC } & $K_{C}=\frac{\tau_{I}}{K_{\mathrm{P}}(\lambda+D)} ; \tau_{I}=\tau+\frac{D^{2}}{2(\lambda+D)}$ and $\tau_{D}=\frac{D^{2}}{2(\lambda+D)}\left[1-\frac{D}{3 \tau_{I}}\right]$ \\
\hline & & & & $\lambda=\max (0.25 D, 0.2 t)$ \\
\hline \multirow[t]{3}{*}{ MS-2C } & $(0.1 \leqslant D / \tau \leqslant 10)$ & PI & Robust-Brambila [2] & $K_{C}=\frac{\tau_{1}+\tau_{2}+D / 2}{K_{\mathrm{P}} D(2 \lambda+1)}$ and $\tau_{I}=\tau_{1}+\tau_{2}+D / 2$ \\
\hline & $(0.1 \leqslant D / \tau<1)$ & PID & IMC-Chien & $K_{C}=\frac{2 \tau}{K_{\mathrm{P}}(\lambda+D)}$ and $\tau_{\mathrm{I}}=2 \tau$ and $\tau_{\mathrm{D}}=0.5 \tau, \lambda=\max (0.25 D, 0.2 t)$ \\
\hline & $(D / \tau \geqslant 1)$ & PID & IMC-Mac & Similar formula as for category $2 \mathrm{U}$ below with $\xi=1$ \\
\hline \multirow[t]{6}{*}{ MS-2U } & $(0.1 \leqslant D / \tau \leqslant 10)$ & PI & Robust-Brambila & $K_{C}=\frac{2 \xi \tau+D / 2}{K_{\mathrm{P}} D(2 \lambda+1)}$ and $\tau_{I}=2 \xi \tau+D / 2$ \\
\hline & $(0.1 \leqslant D / \tau<1)$ & PID & IMC-Chien & $K_{C}=\frac{2 \xi \tau}{K_{\mathrm{P}}(\lambda+D)}$ and $\tau_{\mathrm{I}}=2 \xi \tau$ and $\tau_{\mathrm{D}}=0.5 \tau / \xi$ \\
\hline & \multirow[t]{4}{*}{$(D / \tau \geqslant 1)$} & \multirow[t]{4}{*}{ PID } & \multirow[t]{4}{*}{ IMC-Mac } & $K_{C}=\frac{\tau_{I}}{K_{\mathrm{P}}(2 \lambda+D)}$ and $\tau_{I}=2 \xi \tau-\frac{2 \lambda^{2}-D^{2}}{2(2 \lambda+D)}$ and \\
\hline & & & & $\tau^{2}-\frac{D}{6(2 \lambda+D)}$ \\
\hline & & & & $\tau_{I}$ \\
\hline & & & & $\lambda=\max (0.25 D, 0.2 t)$ \\
\hline
\end{tabular}

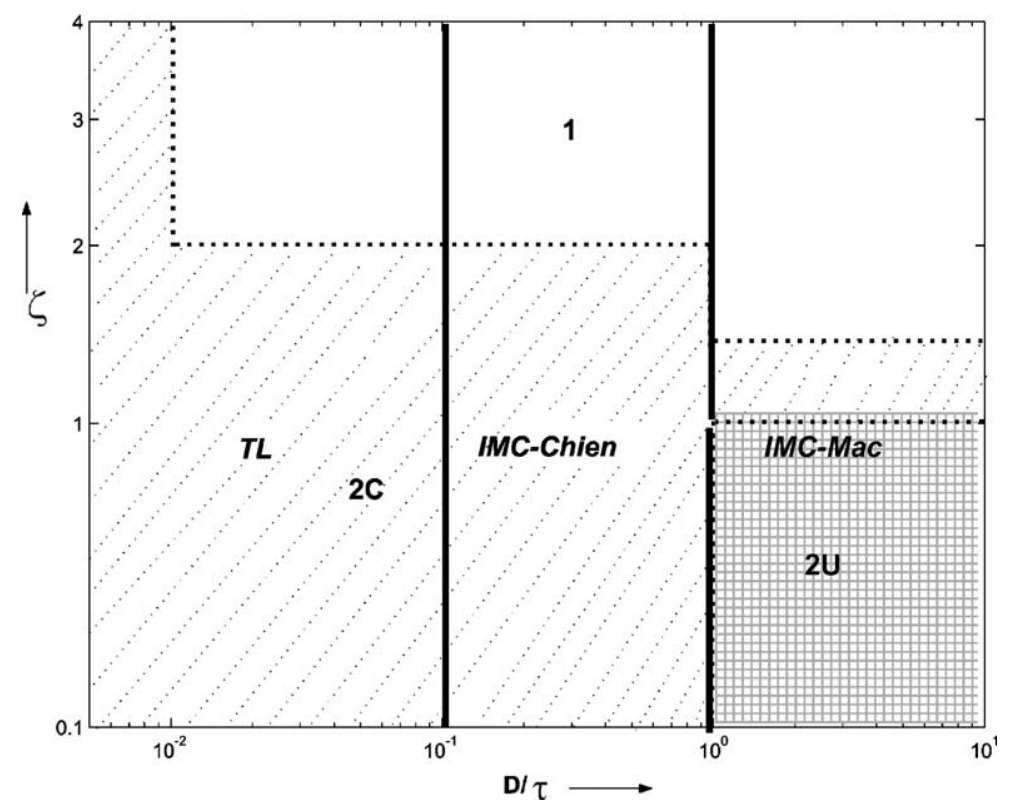

Fig. 13. Recommendations of PID-tuning rules for process with different parameter values (solid line is for tuning region and shaded portion is for selection of model structures).

methods for SOPDT systems are reported in literature. It is suggested that PID controller with IMC-Chien (for processes with $0.1<D / \tau \leqslant 1$ ) tuning or IMC-
Maclaurin [6] (for processes with $D / \tau>1$ ) is suitable for MS-2U type of model structures as it gives better performance. The tuning formula is presented in Table 5. 
Table 6

PID controller parameters and integral absolute error values for the examples

\begin{tabular}{|c|c|c|c|c|c|c|c|c|c|c|c|}
\hline \multirow[t]{2}{*}{ Example } & \multirow{2}{*}{$\begin{array}{l}\text { True } \\
\text { process }\end{array}$} & \multirow{2}{*}{$\begin{array}{l}\text { Identified } \\
\text { process }\end{array}$} & \multicolumn{5}{|c|}{ Proposed tuning rule } & \multicolumn{4}{|c|}{ TL tuning rule } \\
\hline & & & $\begin{array}{l}\text { Tuning } \\
\text { rule }\end{array}$ & $K_{c}$ & $\tau_{i}$ & $\tau_{D}$ & $\begin{array}{l}\text { IAE }^{\mathrm{a}} \\
\text { Servo/Load }\end{array}$ & $\mathrm{Kc}$ & $\tau_{i}$ & $\tau_{D}$ & $\begin{array}{l}\text { IAE } \\
\text { Servo/Load }\end{array}$ \\
\hline Ex-1 & $\frac{1.0 \mathrm{e}^{-0.01 s}}{s^{2}+6.0 s+1}$ & $\frac{1.106 \mathrm{e}^{-0.0022 s}}{(1.066 s+1)^{2}}$ & TL & 247.49 & 0.6094 & 0.0440 & 0.11060 .0025 & 247.49 & 0.6094 & 0.0440 & 0.1106 \\
\hline Ex-2 & $\frac{1.0 \mathrm{e}^{-1.0 s}}{s^{2}+0.4 s+1}$ & $\frac{5.3954 \mathrm{e}^{-1.1566 s}}{(1.2239 s+1)^{2}}$ & IMC-Chien & 0.3138 & 2.4478 & 0.6119 & 7.80398 .3665 & 0.2215 & 14.696 & 1.0603 & 31.442847 .1102 \\
\hline Ex-3 & $\frac{1.0 \mathrm{e}^{-3.0 s}}{s^{2}+6 s+1}$ & $\frac{1.1901 \mathrm{e}^{-3.062 s}}{7.3552 s+1}$ & IMC-Mac & 1.5551 & 8.3894 & 0.9083 & 5.39515 .3947 & 1.4281 & 22.957 & 1.6563 & 13.332515 .0904 \\
\hline Ex-4 & $\frac{1.0 \mathrm{e}^{-7.0 s}}{s^{2}+10 s+1}$ & $\frac{1.036 \mathrm{e}^{-7.03 s}}{10.4411 s+1}$ & IMC-Mac & 1.3922 & 13.151 & 2.2271 & 10.1049 .443 & 0.9814 & 47.548 & 3.4306 & 23.612431 .4234 \\
\hline Ex-5 & $\frac{1.0 \mathrm{e}^{-7.0 s}}{s^{2}+0.4 s+1}$ & $\frac{1.05 \mathrm{e}^{-6.8902 s}}{1.033 s^{2}+0.41 s+1}$ & IMC-Mac & 0.2230 & 2.4199 & 0.2569 & 11.42412 .944 & 0.2816 & 36.641 & 2.6437 & $U^{\mathrm{b}} U$ \\
\hline Ex-6 & $\frac{1.0 \mathrm{e}^{-10.0 s}}{s^{2}+1.6 s+1}$ & $\frac{1.0 \mathrm{e}^{-10.0 s}}{s^{2}+1.6 s+1}$ & IMC-Mac & 0.3011 & 4.5167 & 0.6780 & 15.52615 .516 & 0.5617 & 50.619 & 3.6522 & $\mathrm{U} U$ \\
\hline
\end{tabular}

${ }^{a}$ IAE for load changes in italics.

${ }^{\mathrm{b}} \mathrm{U}$ is unstable.

\subsection{Closed-loop study}

After identifying the proper model structure, a suitable tuning rule for PID controller is found out for the unknown process under investigation. Then closedloop studies are carried out for (a) set-point and (b) load changes. Here, six examples (mentioned in Table 1) are used for closed-loop performance. Naturally, from Table 5, it is suggested that examples 1 and 2 (MS-2C) need TL and IMC-Chien, respectively as $D / \tau \leqslant 1$, whereas other examples (ex-3 to ex-6) require IMCMac tuning. Calculated PID parameters and IAE values
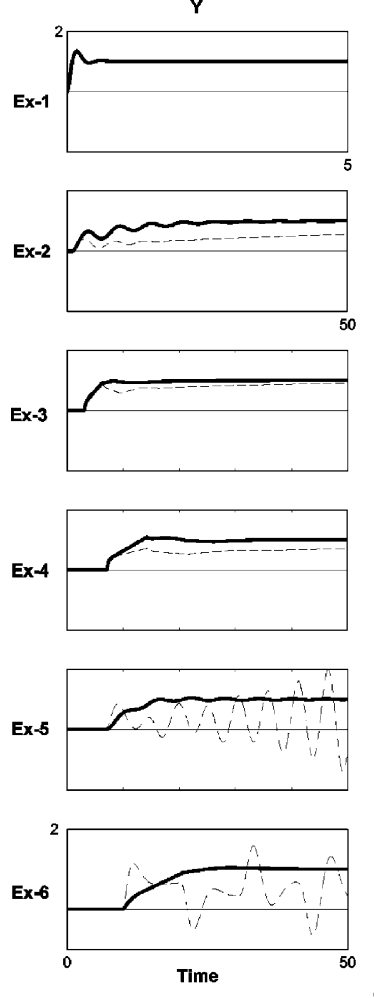
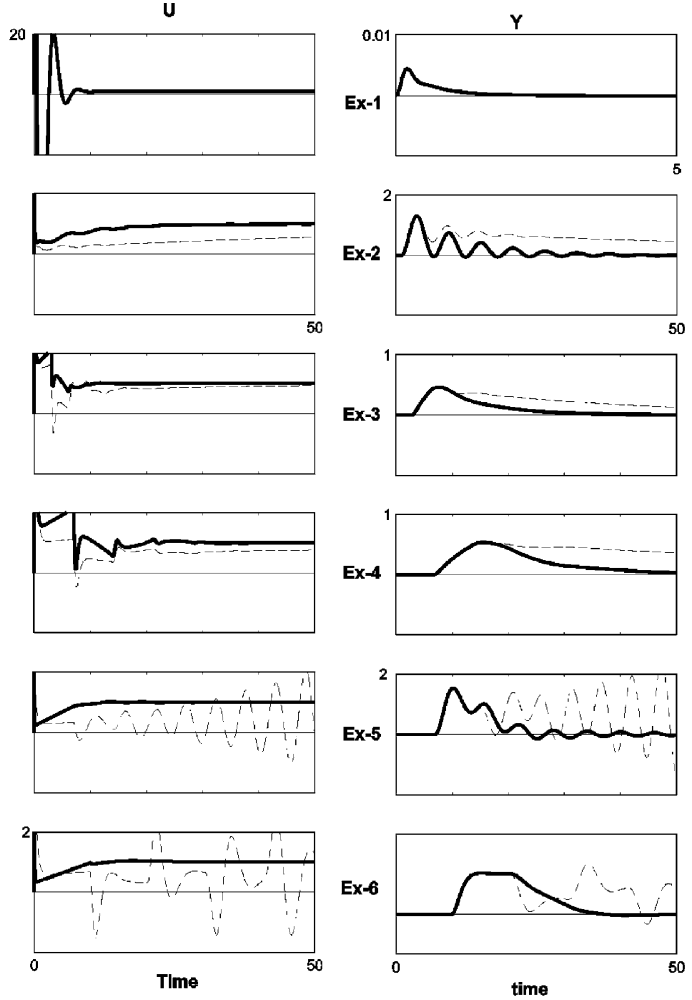
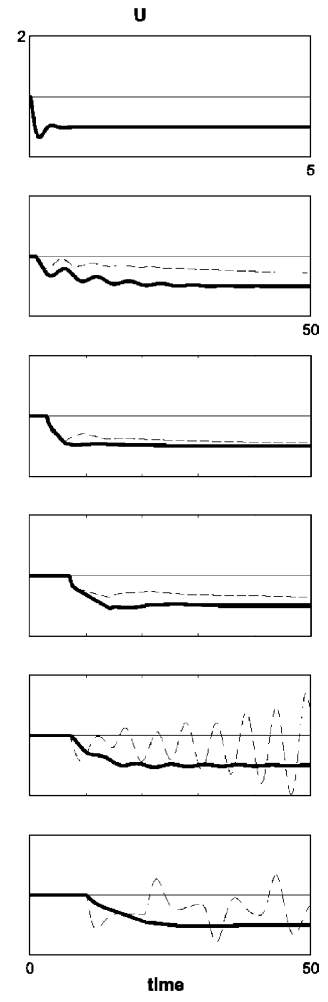

Fig. 14. Closed-loop responses of processes (true processes) with different PID-tuning rules (tuning rules are derived from identified model structures) (dotted line is TL, solid thick line is for IMC-Mac/IMC-Chien) (a) set-point change (b) load disturbance. 
are shown in Table 6. All the PID-controller tuning parameters were calculated based on identified process model parameters (for the examples considered for study). These controllers are implemented on PID2 structure (with $\alpha=0.1$ ).

$K=\mathrm{PID} 2=K_{c}\left(1+\frac{1}{\tau_{i} S}+\frac{\tau_{D} S}{\alpha \tau_{D} s+1}\right)$

In each case, simulation was carried out on true process, from time, $t=0$ to $t=50 \mathrm{~s}$ with set-point $=1.0$. From Fig. 14, we observe closed-loop responses (column $Y$ ) and corresponding controller outputs (column $U$ ), for six different examples undertaken for study. In this figure, dotted line represents response with $\mathrm{ZN}$ tuning rule and thick solid line denotes tuning with IMC-Mac (except IMC-Chien for examples 1 and 2). (ranges of $Y$ axis and $U$ axis are between -2 and 2 where as that of time axis is from 0 to 50 unless mentioned in the figure otherwise). Response time of example-1 is very fast. It can be clearly seen that, examples 1 and 2 (belong to MS-2C) give IAE of 0.1106 and 7.8039 with TL and IMC-Chien for set-point change and IAE of 0.0025 and 8.3665 for load change. The corresponding IAE values with TL tuning are 0.1106 and 31.4428 for set-point and 0.0025 and 47.1102 for load change, examples 3 and 4 (belong to MS-1) yield IAE of 5.3951 and 10.1041 with IMC-Mac for set-point change, where as with TL tuning, these values are 13.3325 and 23.6124, respectively. Again, examples 5 and 6 (belong to MS-2U) give IAE values of 11.424 and 15.526 with IMC-Mac for set-point case but yield unstable response with TL tuning. IMCMac tuning rule yields smoother and faster response. Hence, the performance of this tuning rule is more satisfactory over TL, especially for larger $D / \tau$ cases. The performance of IMC-Mac tuning rule can be further improved (by reducing IAE values) by using optimum tuning parameter, $\lambda$ values.

\section{Conclusion}

Shapes of relay response curves of SOPDT systems are studied here to get a guess on the order and type of unknown process. This method of system identification (Fig. 1) is simple and will be of much industrial use. A single relay feedback test is carried out on unknown system. If the responses are symmetric (in case of asymmetric signals, we may have to do output bias relay adjustment for load or adjustment through hysteresis, at initial stage, for measurement noise) then shapes of the relay response curves guide us to guess on the class/category of the unknown process. Based on three different shapes, three categories of model structures are formulated. Analytical expressions for the relay re- sponse of these categories along with boundary conditions are helpful to identify the model parameters exactly. With the help of analytical expressions for RFB response, limit cycle data and some initial conditions, equations are derived to find out exact model parameters. Classification of systems (Fig. 5) gives a useful hint about the correct model structures of an unknown process. From the model structure and parameter values, we are able to apply appropriate tuning rules (i.e., TL, IMC-Chien and IMC-Mac settings) for better control performance.

\section{References}

[1] K.J. Astrom, T. Hagglund, Automatic tuning of simple regulators with specifications on phase and amplitude margins, Automatica 20 (1984) 645-651

[2] A Brambilla, S. Chen, C. Scali, Robust tuning of conventional controller, Hydrocarbon Process. (1990) 53-58.

[3] R.C. Chang, S.H. Shen, C.C. Yu, Derivation of transfer function from relay feedback systems, Ind. Eng. Chem. Res. 31 (3) (1992) 855-860.

[4] I.-L. Chien, P.S. Fruehauf, Consider IMC tuning to improve controller performance, Chem. Eng. Prog. 86 (10) (1990) 33.

[5] I. Kaya, D.P. Atherton, Parameter estimation from relay autotuning with asymmetric limit cycle data, J. Process Control 11 (2001) 429-439.

[6] Y. Lee, S. Park, M. Lee, C. Brosilow, PID controller tuning for desired closed-loop responses for SI/SO systems, AIChE J. 44 (1) (1998) 106-115.

[7] A. Leva, Autotuning process controller with improved load disturbance rejection, J. Process Control 15 (2) (2005) 223-234.

[8] W. Li, E. Eskinat, W.L. Luyben, An improved auto-tune identification method, Ind. Eng. Chem. Res. 30 (1991) 1530-1541.

[9] W.L. Luyben, Derivation of transfer function for highly nonlinear distillation columns, Ind. Eng. Chem. Res. 26 (12) (1987) 24902495.

[10] W.L. Luyben, Getting more information from relay feedback tests, Ind. Eng. Chem. Res. 40 (20) (2001) 4391-4402.

[11] W.L. Luyben, Effect of derivative algorithm and tuning selection on the PID control of dead-time processes, Ind. Eng. Chem. Res., 40 (2001) 3605-3611.

[12] S. Majhi, D.P. Atherton, Auto-tuning and controller design for processes with small time delays, IEE Proc. Control Theory Appl. 146 (3) (1999) 415-424.

[13] R.C. Panda, C.C. Yu, Analytical expressions for relay feed back responses, J. Process Control 13 (6) (2003) 489-501.

[14] R.C. Panda, C.C. Yu, H.P. Huang, PID tuning rules for SOPDT systems: review and some new result, ISA Trans. 43 (2004) 283 295.

[15] K.K. Tan, Q.G. Wang, T.H. Lee, Finite spectrum assignment control of unstable time delay processes with relay tuning, Ind. Eng. Chem. Res. 37 (4) (1998) 1351-1357.

[16] T. Thyagarajan, C.C. Yu, Improved auto-tuning using shape factor from relay feed back, Ind. Eng. Chem. Res. 42 (2003) 4425 4440 .

[17] Q.G. Wang, C.C. Hang, B. Zou, Low order modeling from relay feedback, Ind. Eng. Chem. Res. 36 (1997) 375-381.

[18] C.C. Yu, Autotuning of PID Controllers, Springer-Verlag, London, 1999. 